a little following the variable to be 11.5. Two other stars lying respectively south and south preceding the variable were assumed to be $\mathbf{I} \mathbf{1 . 2}$ and $\mathbf{1} \mathbf{1} . \mathbf{O}$. There is some little doubt respecting the absolute magnitudes of these comparison stars, as there are considerable differences between the visual and photographic brightness of a number of the stars in this region. But the relative photographic magnitudes were carefully determined. The variable, however, is very much fainter on the photographs than it is in the telescope. On

Hove, I 90 I March 4.
March 3 with a $2 \frac{3}{4}$ inch refractor it appeared considerably brighter than either $\mathrm{BD} .+43^{\circ} 73 \mathrm{I}$ or $\mathrm{BD} .+43^{\circ} .733$ (both 9. I), and certainly not less than $9.0 \mathrm{mag}$. There must be a difference of considerably over a magnitude therefore between the photographic and visual brightness of this star, which is No. 978 of Mr. Espin's list of »Stars with remarkable Spectra». (A. N. 3286). It is there entered as being $R$, and with spectrum of type IV!

\title{
New Variable Star 69.1901.Andromedae.
}

To the list of variables should be added a star not included in the BD. whose approximate place for 1855 is

$$
\text { RA. }=0^{\mathrm{h}} 43^{\mathrm{m}} \cdot 5 \text { Decl }=+33^{\circ} 35^{\prime} \text {. }
$$

On 1900 Oct. 5 this star was $<1 \mathbf{1} \mathbf{m}_{2}$; but on $\mathbf{1 9 0 1}$ Febr. $x 6$ it had risen to $10^{\mathrm{m}} .7$, and on March 10 to $10 \mathrm{~m}_{2}$. These values for its magnitude have been arrived at by

Edinburgh, 2 I East Claremont Street, 190 I March I I.

\section{A. Stanley Williams.}

comparisons with three stars not contained in the BD. whose magnitudes and roughly estimated places for 1855 are as follows:

$\begin{array}{cccc}a & 10^{\mathrm{m}} .4 & 0^{\mathrm{h}} 42^{\mathrm{m}} .8+33^{\circ} 35^{\prime} \\ b & 9.7 & \circ 42.9+3331^{1 / 2} \\ c & 11.2 & \circ 43.0+3330\end{array}$

Thomas D. Anderson.

\section{Nova (3.1901) Persei}

osservata al Piccolo Meridiano di Arcetri.

\begin{tabular}{|c|c|c|c|c|c|c|}
\hline 1901 & $\alpha$ app. & $\delta$ app. & Red. ad & 901.0 & $\alpha 190 \mathrm{r} .0$ & $\delta$ I 90 r.o \\
\hline $\begin{array}{r}\text { ebb. } 25 \\
26 \\
27\end{array}$ & $\begin{array}{r}3^{\mathrm{h}} 24^{\mathrm{m}} 29.79 \\
29.72 \\
29.66\end{array}$ & $\begin{array}{r}+43^{\circ} 34^{\circ} 2.9 \\
3.4 \\
4 \cdot 3\end{array}$ & $\begin{array}{l}-1 \mathrm{~s} 66 \\
-1.64 \\
-1.62\end{array}$ & $\begin{array}{l}-9.6 \\
-9.5 \\
-9.4\end{array}$ & $\begin{array}{r}3^{h} 24^{m} 28.13 \\
28.08 \\
28.04\end{array}$ & $\begin{array}{r}+43^{\circ} 33^{\prime} 53^{\prime \prime} 3 \\
53.9 \\
54.9\end{array}$ \\
\hline
\end{tabular}

Lo splendore di questa stella fu riscontrato in Arcetri, in queste tre belle sere, tanto nel crepuscolo, quanta durante la notte, minore di $\alpha$ Aurigae, e maggiore di $\beta$ Aurigae ed $\alpha$ Persei.

Arcetri:Firenze, rgor Marzo 5.

B. Viaro.

\section{Nova (3.1901) Persei.}

Telegramm aus Jurjew (Dorpat), eingegangen März 2 I, Mittags:

"März 2o Helligkeit Nova Persei nimmt wieder zu. Pokrozesky. «

\section{Beobachtungen von kleinen Planeten.}

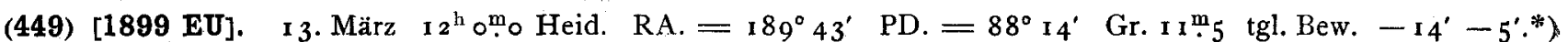
1901 GC. I 3. März I $1^{\mathrm{h}} 38^{\mathrm{m}}$. Heid. RA. $=151^{\circ} 32^{\prime} \mathrm{PD} .=81^{\circ} 7^{\prime}$.

1901 GD. I 3 . März I $1^{\mathrm{h}} 38^{\mathrm{m}}$ 。 Heid. RA. $=152^{\circ} 50^{\prime} \mathrm{PD} .=79^{\circ} 44^{\prime}$.

Heidelberg, I 90 I März I 4 .

M. Wolf.

*) Den Theilnehmern der Centralstelle als »Planet 190I GH vielleicht 449 « telegraphisch mitgetheilt. Die Ephemeride in Veröff. R. I. Nr. I3 ist fehlerhaft. Herr $\mathcal{F}$. Möller hat eine im Anschluss an vorstehende Beobachtung verbesserte Rechning mehreren Sternwarten brieflich zugehen lassen. $K r$.

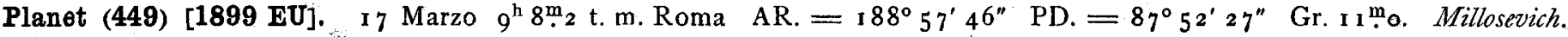

Inhalt zu Nr. 3697-98. A. Antoniazzi. Comete e Pianeti. I. - V. F. Ascarza. Nota sobre la longitud de onda de la raya verde (1474 K) del espectro de la corona solar. 23. - F. Bauschinger. Bahnen der kleinen Planeten (457) bis (463). 23. - E. Millosevich. Quarta parte dell'Effemeride di (433) Eros. 25. - Ch. André. Sur le système formé par la Planète double (433) Eros. 27. - A. Stanley. Williams. New Variable Star 68.1901 Persei. 29. - Th. D. Anderson. New Variable Star 69. I90I Andromedae. 3I - B. Viaro. Nova (3.190I) Persei. 3I. - Nova (3.r9oI) Persei. 31. - M. Wolf. Beobachtungen von kleinen Planeten. 3I. - Millosevich. Planet (449) [1 899 EU]. 3 I. 